\title{
A Non Randomized Controlled Study to Evaluate the Effect of Isotonic Handgrip Exercise on Blood Pressure in Normal Weight and Preobese Healthy Adults
}

\author{
Akash Patel ${ }^{1}$, Nilesh Patel ${ }^{2}$ \\ ${ }^{1}$ MBBS Intern, College Address: Smt. B.K. Shah Medical Institute and Research Centre, Pipalya Road, Vadodara, \\ Gujarat, ${ }^{2}$ WAS Assistant Professor, Department of Physiology S.B.K.S. M.I. \& R.C., Recently Assistant Professor, \\ Department of Physiology, Pramukhswami Medical College, Karamsad, College in Anand
}

\begin{abstract}
Background: The adverse health consequences of overweight and obesity in India leads to higher prevalence of diabetes mellitus and cardiovascular diseases. Also the compliance of people for routine form of exercise for BP control has not been very encouraging due to time, place etc constrains.

Aim: Therefore we conducted a nonrandomised clinical study to determine the short-term effects of isotonic handgrip exercise by using smiley balls on blood pressure in healthy normal weight and overweight adolescents with the objective to find a user friendly exercise which help in reducing blood pressure.

Method: A non randomized clinical study was conducted on 100 young normal-weight and pre-obese adults (50 Boys and 50 Girls) in the age group of 18-25 years. Isotonic handgrip exercise was performed at the rate 20 contractions/minute ( $2 \mathrm{sec}$ contraction/ $1 \mathrm{sec}$ relaxation) at maximal intensity for 10 minutes using smiley ball. Pulse rate and blood pressure parameters were tested at baseline and immediately after exercise in post-exercise recovery period.
\end{abstract}

Result: Statistically significant reduction was observed in systolic blood pressure(SBP) and mean arterial pressure (MAP) in both pre-obese boys and girls groups while pulse pressure \& mean arterial pressure in normal weight girls after exercise regime.

Conclusion: We conclude that the exercise regime under consideration can produce some short-term beneficial effects with respect to blood pressure in especially pre-obese group of adults.

Keywords: Isotonic Handgrip Exercise, Normal weight and pre-obese adults.

\section{Introduction}

It is known that regular dynamic exercise reduces blood pressure (BP) and helps in the prevention of hypertension by various mechanisms such as decrease in sympathetic nerve traffic, potentiation of baroreceptor reflex, decrease inarterialstiffness, increase in total systemic arterial compliance, increase in the release of endothelium-derived nitric oxide, and increase in insulin sensitivity.It has also been reported that an acute bout of dynamic physical exercise involving large muscle mass also results in lowering of BP lasting for 12-16 hoursin the postexercise period, known as postexercise hypotension (PEH). ${ }^{1}$
The prevalence of overweight and obesity among children and adolescents has increased significantly in the developed countries during the past two decades and similar trends are being observed even in the developing world. Targeting adolescent age group for primary prevention can be justified for many reasons. The adverse health consequences of overweight and obesity in India leads to higher prevalence of type 2 diabetes and cardiovascular diseases. ${ }^{2}$

However, despite the strong evidences that recommend regular dynamic exercise involving large muscle mass (briskwalking and cycling) for lowering $\mathrm{BP}$ and prevention of hypertension, the compliance 
of people toward such forms of exercise has not been encouraging owing to various possible reasons such as comorbidconditions (osteoarthritis and coronary artery disease)and other constraints (such astime, space, and economic constraints). ${ }^{3}$

It is, therefore, necessary to design an alternative feasible form of physicalexercise involving a relatively lesser muscle mass, which can reduce BP and increase exercise compliance in the population.

Isotonic handgrip exercise is a simple, cheap, and feasible form of physicalexercise involving relatively lesser muscle mass, which can be performed as perindividual convenience with respect to time and place using simple equipment such as handgrip dynamometer, smiley ball. But, before we prescribe isotonic handgripexercise to the population, it is essential to determine whether isotonichandgrip exercise decreases $\mathrm{BP}$ in post-exercise period as an acute short-termeffect (PEH) and/or over a long term as a training effect. It is also essentialto understand the mechanism underlying such BP-lowering effects of isotonic handgrip exercise. As scant literature is available, which illustrate the role ofisotonic handgrip exercise in lowering blood pressure, We investigated a single bout ofisotonic handgrip exercise for PEH.

\section{Aim \& Objectives of Study}

- To evaluate the effect of isotonic handgrip exercise on blood pressure

- To compare the effect of exercise on normal and preobese healthyadults in both genders.

\section{Material and Method}

- Study Design: A Non randomized controlled study

- Study Population: Medical students of age group 18 to 25 years and both the genders

- Sample size: 100 students (50 girls and 50 boys including normal weight and pre-obese)

\section{- Inclusion criteria:}

1. Age group 18 to 25 years boys and girls

2. BMI -18.5 to 29.9 (normal weight-18.5-24.9; pre-obese -25 to $29.9 \mathrm{~kg} / \mathrm{m} 2)^{2,4}$

\section{Exclusion Criteria:}

1. BMI less than $18.5 \mathrm{~kg} / \mathrm{m} 2$ and more than $30 \mathrm{~kg} / \mathrm{m} 2$

2. Participants who were smokers, athletes and suffering from chronic illness

\section{Method}

Present study was conducted on 100 voluntary participants (50 Boys and 50 Girls) at SBKS Medical Institute and Research Center (SBKS MIRC)after taking approval from Human Research Review Penal (HRRP) of SBKS MI \& RC and Institutional Ethics Committee (SVIEC) of our institute and consent from participants.

Those medical students who were in the age group of 18 to 25 years and had BMI in the range of 18.5 to 29.9 $\mathrm{kg} / \mathrm{m} 2$ were included in study. The body weight (Wt) was measured bare footed to the nearest $0.5 \mathrm{~kg}$ and the height was measured using meter scale without footwear to the nearest $0.5 \mathrm{~cm}$. Body Mass Index (BMI) will be calculated as the weight $(\mathrm{kg})$ divided by thesquare of height (m2). ${ }^{1}$

The study participants were first tested for preexercise(baseline) pulse rate $\&$ blood pressure. This was followed by a bout of isotonic handgrip exercise for 10 minutes. After exercise, again pulse rate \& blood pressure was taken. ${ }^{3}$

Method of Measurement of BP: The blood pressure was measured in the non-dormant arm in the sitting position with arm and back support. The blood pressure was recorded at the interval of $1 \mathrm{~min}$ till the difference between two consecutive blood pressure regarding was $<5 \mathrm{mmHg}$. The average of the two consecutive readings was used for stastical analysis. The systolic and diastolic blood pressure was measured by using digital sphygmomanometer ${ }^{1}$. Pulse pressure (PP) was calculated by using formula: systolic blood pressure (SBP) - diastolic blood pressure (DBP). Mean arterial pressure (MAP) was calculated by formula (Diastolic Pressure $+1 / 3$ Pulse pressure(PP). ${ }^{1}$

Method of Performing Single Bout of Isotonic Handgrip Exercise: The single bout of isotonic handgrip exercise was performed by the dominant hand of the participants using smiley ball for duration of 10 minutes continuously at an intensity of MVC. During the exercise the participant was asked to squeeze the ball for 2 seconds (Contraction phase) followed by release of the ball for 1 second (Relaxation phase) such that a compression cycle rate of 20/min (each cycle had 2 seconds contraction followed by 1 second of relaxation) is achieved. (1) Exercise was stopped if the heart rate rises above $85 \%$ MHR or Blood Pressure rises above 180/110 mmHg..$^{1,3}$ 
Statistical Analysis: Mean and Standard Deviation of the study variables were calculated for Pre-exercise baseline and Immediate Post-Exercise Period. Student's Paired t-test was used to study if any significant differences in study variables were observed between the Pre-exercise and the post-exercise periods. $\mathrm{P}$ value $<$ 0.05 was considered as significant.

\section{Ethical Issues}

No ethical issue in this research project because of

- Intervention was simple handgrip exercise in the study

\section{Observations:}

Table 1: Subject Characteristics

\begin{tabular}{|l|c|c|c|c|}
\hline & \multicolumn{2}{|c|}{ Boys(n=50) } & Girls (n=50) \\
\hline & Normal (n=27) & Preobese(n=23) & Normal (n=28) & Preobese(n=22) \\
\hline Age $($ Yrs) & $21.6 \pm 1.63$ & $20.7 \pm 1.3$ & $20.4 \pm 0.64$ & $20.6 \pm 0.94$ \\
\hline Weight $(\mathrm{Kg})$ & $63 \pm 7.07$ & $77.83 \pm 8.37$ & $50 \pm 5.94$ & $57.4 \pm 4.16$ \\
\hline Height $(\mathrm{Cm})$ & $172.3 \pm 9.08$ & $170.8 \pm 11.3$ & $158 \pm 6.34$ & $152 \pm 9.45$ \\
\hline BMI $\left(\mathrm{Kg} / \mathrm{M}^{2}\right)$ & $21.1 \pm 1.5$ & $27.04 \pm 1.41$ & $20.02 \pm 1.49$ & $26.55 \pm 1.4$ \\
\hline Values indicate Mean $\pm \mathrm{SD}^{*}$ Indicates significant difference between groups. P value $<0.05$ & \\
\hline
\end{tabular}

Table 2: Short-term Effects of Single Bout of Isotonic Handgrip Exercise on Blood pressure in boys

\begin{tabular}{|l|c|c|c|c|c|c|}
\hline & \multicolumn{3}{|c|}{ Normal weight } & \multicolumn{3}{c|}{ Preobese } \\
\hline & Baseline & Post-exe & p-Value & Baseline & Post-exe & p-Value \\
\hline Pulse Rate & $82.27 \pm 6.56$ & $84 \pm 6.9$ & 0.35 & $83.63 \pm 5.34$ & $86 \pm 6.5$ & 0.184 \\
\hline SBP & $122.4 \pm 4.89$ & $119 \pm 6.37$ & 0.087 & $129.1 \pm 5.67$ & $126 \pm 6.32$ & $0.017^{*}$ \\
\hline DBP & $76.6 \pm 5.87$ & $75.2 \pm 5$ & 0.35 & $81.4 \pm 7.33$ & $78.2 \pm 6.89$ & 0.135 \\
\hline PP & $45.75 \pm 5.58$ & $44 \pm 7.6$ & 0.34 & $47.75 \pm 5.23$ & $47 \pm 4.7$ & 0.612 \\
\hline MAP & $92 \pm 4.9$ & $89.8 \pm 4.16$ & 0.081 & $97 \pm 6.4$ & $94 \pm 6.33$ & $0.019 *$ \\
\hline Values indicate Mean $\pm \mathrm{SD}^{*}$ Indicates significant difference between groups. P value $<0.05$ & & \\
\hline
\end{tabular}

Table 3: Short-term Effects of Single Bout of Isotonic Handgrip Exercise on Blood pressure in Girls

\begin{tabular}{|l|c|c|c|c|c|c|}
\hline & \multicolumn{3}{|c|}{ Normal weight } & \multicolumn{2}{c|}{ Preobese } \\
\hline & Baseline & Post-exe & p-Value & Baseline & Post-exe & p-Value \\
\hline Pulse Rate & $79.79 \pm 9.35$ & $81.3 \pm 6.93$ & 0.48 & $79.83 \pm 8.9$ & $82.5 \pm 4.45$ & 0.226 \\
\hline SBP & $116 \pm 9.43$ & $105.9 \pm 8.66$ & 0.64 & $115 \pm 7.44$ & $106.6 \pm 8.75$ & $0.002 *$ \\
\hline DBP & $71.6 \pm 6.4$ & $71 \pm 5.76$ & 0.709 & $74.7 \pm 6.99$ & $75.3 \pm 7.11$ & 0.784 \\
\hline PP & $44 \pm 11$ & $35 \pm 7.3$ & $0.001^{*}$ & $40 \pm 9.7$ & $31 \pm 10$ & $0.005^{*}$ \\
\hline MAP & $86.25 \pm 5.68$ & $82.7 \pm 5.94$ & $0.024^{*}$ & $106.6 \pm 8.75$ & $85.7 \pm 5.98$ & $0.045^{*}$ \\
\hline \multicolumn{7}{|l|}{ Values indicate Mean $\pm \mathrm{SD}^{*}$ Indicates significant difference between groups. P value $<0.05$} \\
\end{tabular}


Table 1 shows subject characteristics age in yrs, weight $(\mathrm{Kg})$, height $(\mathrm{Cms})$ and $\mathrm{BMI}\left(\mathrm{Kg} / \mathrm{M}^{2}\right)$ in both genders. As depicted in Table 2 and Table 3, in comparison to the pre-exercise baseline condition, systolic blood pressure(SBP), mean arterial pressure(MAP) in Preobese groups of both genders were found to be significantly different in the Post-Exercise period. Table 3 shows also significant reduction in pulse pressure(PP) and mean arterial pressure (MAP) in normal weight girls.

\section{Discussions}

The current study indicates that Isotonic Handgrip Exercise performed for 10 minutes at an intensity of MVC at a Compression cycle of 20 contractions per minute can produce post-exercise hypotension (PEH) in preobese adults as compared to normal weight adults specially in girls.

PEH has been well documented in humans with both borderline hypertension and hypertension. However, its occurrence in normotensive humans is inconsistent because of lesser magnitude than in hypertensive individuals and compensatory mechanisms such as the Baroreflex, which are activated in normotensive. ${ }^{5} \mathrm{JR}$ Macdonald et al had found no difference in magnitude of hypotension following $30 \mathrm{~min}$ of cycle ergometry at $50 \%$ and $75 \% \mathrm{VO}_{2}$ peak in normotensive volunteers. ${ }^{5}$ In contrast Pescatello et al were unable to document PEH in a normotensive. ${ }^{6}$ These findings show that the effect of dynamic exercise on blood pressure in normotensive subjects is less clear. Our study has been conducted on healthy young normalweight adolescents aged 18-25 yrs and this could be possible reason for no significant results after a single exercise session.

Victor Ronald et al. found no significant difference in mean arterial pressure after rhythmic handgrip exercise at 10, 30 and $50 \%$ of MVC and mild two-arm cycling at 0 to 20 watt, but at 40 and 60 watt intensity two arm cycling shows significant difference in arterial pressure response as compared to baseline values in recovery period. ${ }^{7}$ Present study shows significant difference in systolic blood pressure and mean arterial pressure in Preobese adults after a single bout of 10 minutes isotonic handgrip exercise at maximal intensity.

Nilesh Patel et al. study showed no significant difference in blood pressure due to normotensive adolescents and lower intensity isotonic handgrip exercise with more relaxation as compared to contractions. ${ }^{1}$ Current study also has no significance result in normal weight group but has significant difference in Preobese group due to higher intensity exercise with more contraction duration as compared to relaxation during exercise session.

A recent meta-analysis of 18 prospective cohortshas shown elevated the risk of cardiovascular disease (CVD) by 1.55 (relative risk, RR), coronary heartdisease (CHD) by 1.50 , and stroke by 1.71 in pre-hypertension group compared to normotensive group. It is one of the most common modifiable risk factors in CVD. Mean reduction of $3.0 \mathrm{mmHg}$ in SBP could reduce mortality from CHD by $6 \%$ and from stroke by $9 \%$. Another subanalysis for healthy and hypertensive studies showed mean reduction of $4.03 \mathrm{mmHg}$ in SBP and of $2.96 \mathrm{mmHg}$ in DBP. ${ }^{8}$ The current study also reported significant reduction in SBP and MAP in Preobese groups in both genders.

Isotonic forearm exercise produces less of demand on heart than continuous isometric exercise. Marjorie et al. found that cardiovascular response produced by isotonic exercises is intensity dependant and exercise sessions which includes relaxation between contractions shows lower cardiovascular response. ${ }^{9}$ Many studies done as rhythmic handgrip exercises show significant rise in Muscle Sympathetic Nerve Activity (MSNA) and blood pressure during exercise sessions of different intensity but post-exercise response is less significant. ${ }^{7}$ In the present study, we have used protocol for isotonic handgrip exercise with less relaxation between contractions and which is probably responsible for the exercise to produce cardiovascular changes in postexercise period specially in preobese adults.

Three mechanisms are thought to be responsible for the neural cardiovascular modulation during voluntary muscle contractions: an activation of higher brain centers ("central command") as well as reflex activity primarily involving inputs from chemo- and mechanoreceptor ("muscle metaboreflex") and baroreceptor afferents ("baroreflex"). The influence of each mechanism on the heart rate and blood pressure response to exercise depends on factors like recruited muscle mass, muscle fiber type, exercise intensity and the exercise mode. ${ }^{\mathbf{1 0}}$

When oxygen delivery to active skeletal muscle is insufficient to meet the metabolic demands during dynamic exercise, metabolites (e.g., lactic acid, adenosine, potassium, diprotonated phosphate, and arachidonic acid products, among others) accumulate within the active muscle and stimulate group III and 
IV afferent neurons. These sensory neurons project to the medulla oblongata and their activity elicits reflex increases in sympathetic nerve activity and systemic blood pressure in an effort to enhance blood flow to the ischemic muscle. Termed the muscle metaboreflex, this response is thought to provide important functional links between metabolism in active muscles and central hemodynamics during exercise. Our results show that increasing workload shifts the muscle metaboreflex threshold to higher blood flow levels and therefore reduces the change in blood flow necessary toelicit a reflex pressor response. ${ }^{11}$

\section{Conclusion}

Present study reveals that a single bout of an isotonic handgrip exercise performed by dominant hand at an intensity of MVC using a smiley ball with contraction frequency of 20/minute for 10 minutes can produce post-exercise hypotension into the post-exercise period. However, further studies are required to determine if such exercise form can produce $\mathrm{PEH}$ in the hypertensive population and to study the long-term effects of training on blood pressure with such form of exercise in larger population.

Study limitations and Future Perspective: A major limitation of the study was that the participants were also normotensive individuals. Thus, it is essential to study the effect in Prehypertensive and hypertensive population. It is also required to determine if $\mathrm{PEH}$ can be produced by lowering the intensity, contraction cycle rate and duration of exercise. And finally, it is essential to study the effects of isotonic handgrip exercise training on blood pressure and cardiovascular autonomic functions in larger groups.

Ethical Clearance: Taken From Sumandeep Vidyapeeth Institutional Ethics Committee (SVIEC).

Source of Funding: Self

Conflict of Interest: Nil

\section{References}

1. Nileshkumar H Patel, Wasim Shaikh, Sushil Kumar Singh; Can isotonichandgrip exercise cause postexercise hypotension in healthy adolescents? International Journal of Medical Science and Public Health | 2015 | Vol 4 | Issue 11;1580-1583.

2. Anand Krishna Gorantla, Murari Pradeep Kumar; A cross sectional study on association between overweight and obesity in adolescent population; Int J Community Med Public Health. 2017 Jan;4(1):115-119.

3. Nileshkumar H Patel, Hasmukh D Shah, Wasim A Shaikh, Sushil K Singh; Short term effects of isotonic handgrip exercise on cardiovascular autonomic reactivity in healthy young adoloscents;National Journal of Community Medicine|Volume 6!Issue 2'Apr - Jun 2015;390-393.

4. K. Park; Textbook of Preventive and Social Medicine; 24 ${ }^{\text {th }}$ Edition; Epidemiology of chronic non communicable diseases and conditions;pp 335370.

5. JR MacDonald, JD MacDougall and CD Hogben. The effects of exercise duration on postexercise hypotension. Journal of Human Hypertension 2000; 14: 125-129.

6. Linda S. Pescatello, Ann E. Fargo, Charles N. Leach Jr. and Herbert H. Scherzer. Short-term effect of dynamic exercise on arterial blood pressure. Circulation 1991; 83: 1557-1561.

7. Ronald G. Victor, Douglas R. Seals, and Allyn L. Mark. Differential control of heart rate and sympathetic nerve activity during dynamic exercise; insight from intraneural recordings in humans. J. Clin. Invest. 1987; 79: 508-516.

8. SonuPunia, SivachidambaramKulandaivelan, Varun Singh,and Vandana Punia; Effect of Aerobic Exercise Training on Blood Pressure in Indians: Systematic Review; International Journal of Chronic Diseases;Volume 2016;Article ID 1370148; 8 pages.

9. Marjorie Greer, Susan Dimick and Stephanie Burns. Heart rate and blood pressure response to several method of strength training. PHYS THER. 1984; 64:179-183.

10. Matthias Weippert, Kristin Behrens, Annika Rieger, Regina Stoll, Steffi Kreuzfeld; Heart Rate Variability and Blood Pressure during Dynamic and Static Exercise at Similar Heart Rate Levels; December 2013 | Volume 8 | Issue 12;pp.

11. Ichinose M, Delliaux S, Watanabe K, Fujii N, NishiyasuT.;Evaluation of muscle metaboreflex function through graded reduction in forearm blood flow during rhythmic handgrip exercise in humans;Am J Physiol Heart Circ Physiol301: H609-H616, 2011. 